\title{
A rare case of racemic solid solution in mercury complexes
}

Zahrasadat Momenzadeh Abardeh ${ }^{1}$, Alireza Salimi ${ }^{1}$, Jered Garrison ${ }^{2}$

${ }^{1}$ Ferdowsi University Of Mashhad, Mashhad, Iran, Islamic Rep., ${ }^{2}$ Department of Pharmaceutical Sciences, University of Nebraska Medical Center, Nebraska, United States

E-mail: zahramomen9064@yahoo.com

Trying of crystal engineering is to design the molecular building blocks can be packed in a manner guided to obtain the desired structural characteristics. In this work, two new complexes of $([\mathrm{Hg} 2 \mathrm{Cl} 4 \mathrm{~L} 2(\mathrm{OH} 2)], 1,[\mathrm{Hg} 2 \mathrm{Br} 4 \mathrm{~L} 2(\mathrm{OH} 2)], 2 \mathrm{were}$ designed and synthesized including the racemic carboxamide ligand $(\mathrm{L})$. These compounds were characterized using FT-IR, CHNS elemental analyses and single crystal X-ray diffraction. The crystal structure analysis showed that the complexes were crystallized in the triclinic crystal system, space group Pĩ. The repetitive molecular unit of titled compounds was included the tetrameric form of mercury (II) chloride and bromide, respectively, which was connected to four ligand molecules. These new complexes were crystallized as the racemic solid solution (pseudoracemate) that is a very rare state of racemic mixture with a probability of less than $1 \%$ in the coordination compounds. In this case, enantiomeric forms of ligand are distributed randomly in the crystal structures by various enantiomeric disorders. In the aforementioned compounds, the $\mathrm{R}$ and $\mathrm{S}$ enantiomer forms of ligand in two terminal (LT) and medium (LM) positions showed enantiomeric disorder with different occupancy factors which led to four states of complex tetrameric units for 1 and two states for 2, simultaneously. Interestingly, the investigation of non-covalent interactions showed that the specific hydrogen bonding has an important role in the control of enantiomeric disorder forms in the title pseudoracemate structures. Interestingly, the chiral hydrogen atom of tetrahydrofuran ring is located in the suitable direction relative to halogen atom to from the interamolecular hydrogen bond interaction.

[1] Chulvi. K. et al. (2015) Crystal Growth Des. 15, 354-358.

[2] Custelcean. R. et al. (2005) Crystal Growth Des. 5, 2277.

[3] Huang. J. et al. (2006) J. Am. Chem. Soc. 128, 11985-11992.

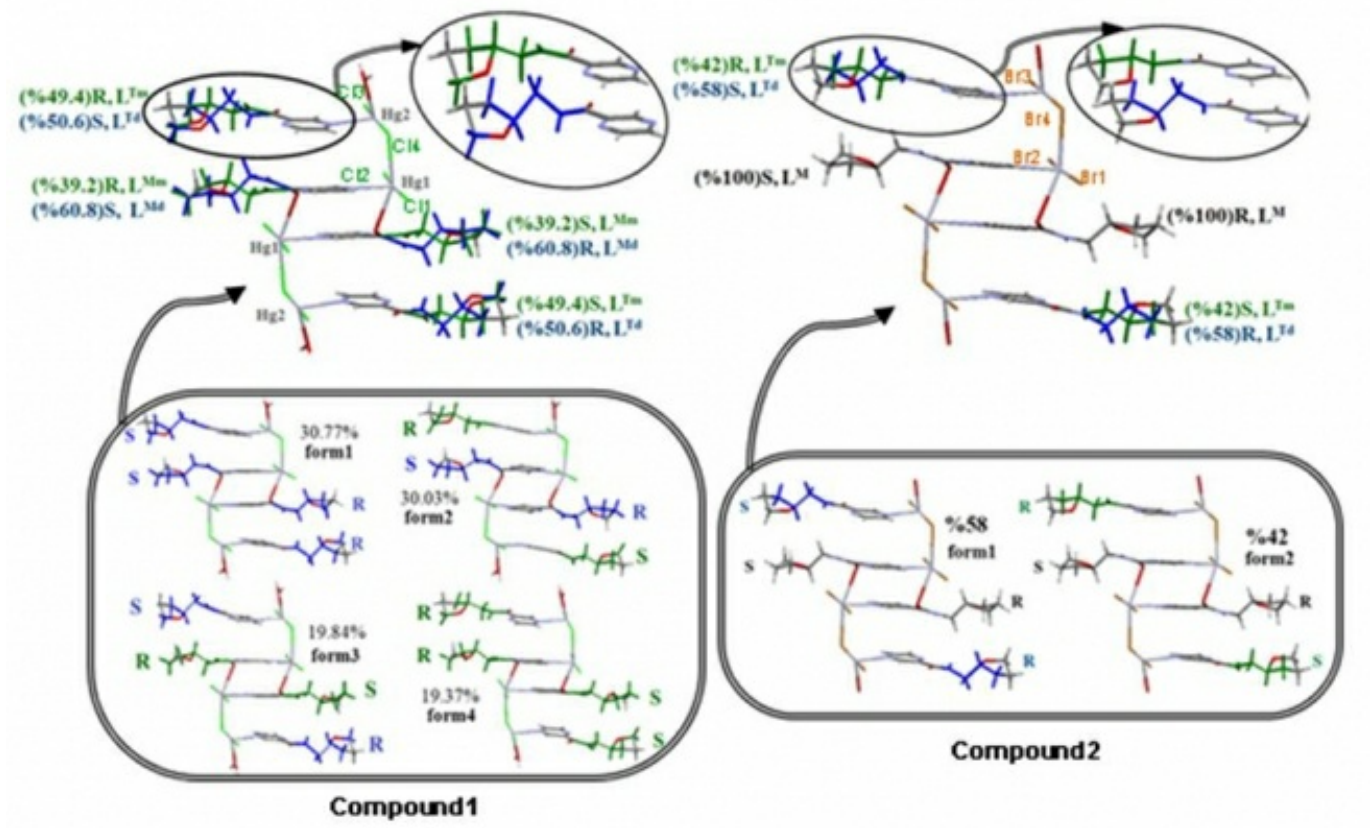

Keywords: crystal engineering, racemic solid solution, enantiomeric disorder 